\title{
Comparison of the Image Rejection between the Passive and the Gilbert Mixer
}

\author{
Wang, Xiaoyan; Andreani, Pietro
}

Published in:

Proceedings of ISCAS '04

Link to article, DOI:

10.1109/ISCAS.2004.1328358

Publication date:

2004

Document Version

Publisher's PDF, also known as Version of record

Link back to DTU Orbit

Citation (APA):

Wang, X., \& Andreani, P. (2004). Comparison of the Image Rejection between the Passive and the Gilbert Mixer. In Proceedings of ISCAS '04 (Vol. 1, pp. 968-971). IEEE. https://doi.org/10.1109/ISCAS.2004.1328358

\section{General rights}

Copyright and moral rights for the publications made accessible in the public portal are retained by the authors and/or other copyright owners and it is a condition of accessing publications that users recognise and abide by the legal requirements associated with these rights.

- Users may download and print one copy of any publication from the public portal for the purpose of private study or research.

- You may not further distribute the material or use it for any profit-making activity or commercial gain

- You may freely distribute the URL identifying the publication in the public portal

If you believe that this document breaches copyright please contact us providing details, and we will remove access to the work immediately and investigate your claim 


\title{
COMPARISON OF THE IMAGE REJECTION BETWEEN THE PASSIVE AND THE GILBERT MIXER
}

\author{
Xiaoyan Wang and Pietro Andreani \\ Center for Physical Electronics, Ørsted · DTU \\ Technical University of Denmark, Denmark
}

\begin{abstract}
This paper presents a comparison of the image rejection between Gilbert mixer and the passive mixer. A simple model for mixers is set up, and the image rejection performance of passive and Gilbert mixer is analyzed based on it. Simulations and calculations were done to compare the image rejection of the two mixers. The results show that the Gilbert mixer, comparing with the passive one, shows a stronger rejection to the amplitude error of the quadrature signals at its input.
\end{abstract}

\section{INTRODUCTION}

The image rejection transceiver has been an promising solution for higher level integration of RF ICs, since it eliminates the image rejection filter (IR filter). One of the prerequisite of implementing this transceiver is the availability of the accurate quadrature local oscillator (LO) signals. The accuracy of the quadrature is usually evaluated by means of the image band rejection (IBR) ratio, using a single side-band (SSB) up-conversion circuit.

Different solutions at the circuit level have been proposed to improve the quadrature of the LO signal. A number of so-called series quadrature voltage-controlled oscillators (QVCOs) were proposed [1] [2], presenting an IBR, ranging from 40-50dB, and phasenoise Figure-of-Merit (FoM) of 184dB. A quadrature mixer [3] has also been proposed, to correct the amplitude/phase error.

Theoretical works have also been done, to analyze the amplitude/phase error generated in active mixer [4]. The results show that as the amplitude of the LO signal increases, the impact of the mismatches in the Gilbert cell on the image rejection decreases. In another work [5], the image rejection is analyzed as a function of the phase and amplitude mismatches for Hartley and Weaver structures, showing that the IBR vs. phase/amplitude error function differs when the amplitude of the LO signal changes. It was not mentioned which kind of mixer was used in the structure. A detailed comparison between the Gilbert mixer and the passive mixer has been presented in [6], focusing on the conversion gain, noise figure, linearity and power consumption.

In this paper, the comparison of the image rejection performance between the passive and the Gilbert mixer is presented. In section II, a simple model used to analyze the image rejection of mixers is described, and a general formula used to calculate the IBR is derived based on the model. The image rejection performances of the Gilbert mixer and the passive mixer are analyzed with the model and the formula. Comparison between the two mixers are done in section III, supported by simulation and calculation results. The conclusion is drawn in the last section.

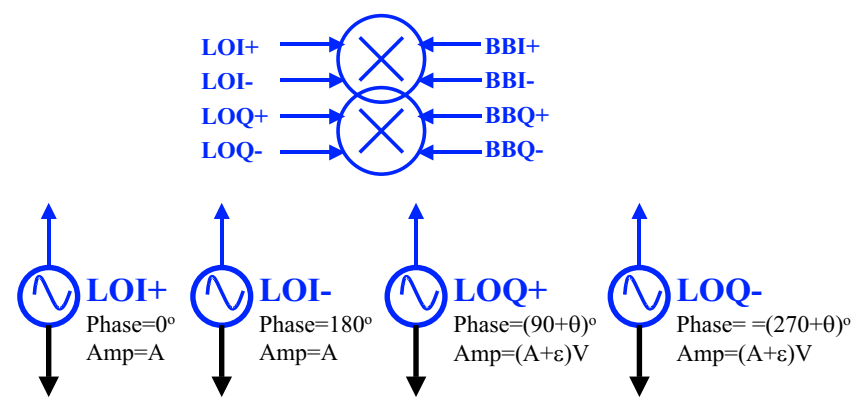

Figure 1: Schematic of Gilbert mixer simulation.

\section{THEORETICAL ANALYSIS OF THE IMAGE REJECTION IN MIXERS}

\subsection{Modelling of Mixers}

The image rejection of the mixer can be easily simulated by using a SSB up-conversion circuit. In order to be able to control the input amplitude/phase error of the input signal, sinusoidal voltage sources are used to generate the LO and the baseband signal, as shown in Fig.1. For the convenience of the analysis, it is assumed that all the input amplitude/phase error come from the LO signal, and the baseband signals are in perfect quadrature.

The input amplitude/phase error, translated into IBR, can be calculated by the following equation, which is a transform of the equation in [7],

$$
I B R=10 \log \frac{1}{\frac{\left(\frac{\epsilon}{A}\right)^{2}+\theta^{2}}{4}},
$$

where $\epsilon$ is the the amplitude error, $\theta$ represents the phase error in radians, and $\mathrm{A}$ is the amplitude of the LO signal. Here, it is assumed that $\epsilon \ll A$ and $\theta \ll 1 \mathrm{rad}$. In this paper, we focus only on the input amplitude error and ignore the phase error. Therefore, the phase error $\theta$ is set at 0 .

The baseband signal can be simplified as a DC voltage (for passive mixer) or a DC current (for Gilbert mixer) since the frequency of it is much lower than the LO frequency. The output signal of the mixer, $V_{\text {out }}$, is an upconverted signal at the same frequency as the LO signal. As the LO signal changes, $V_{\text {out }}$ may change. The first harmonic of the output signal can be calculated by,

$$
V_{1 s t}=\frac{1}{T} \int_{0}^{T} V_{\text {out }}\left(V_{L O}\right) \cdot e^{-j \omega t} d t
$$




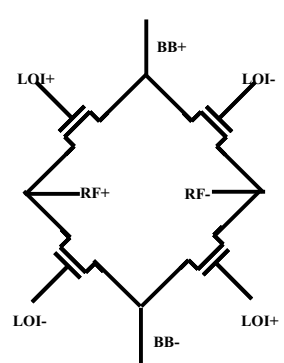

(a)

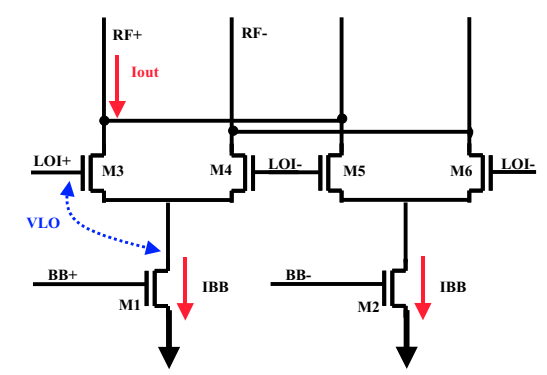

(b)

Figure 2: a).Schematic of passive mixer; b).Schematic of active mixer.

where $\omega=\frac{2 \pi}{T}$, and $\mathrm{T}$ is the period of the LO signal. The function $V_{\text {out }}\left(V_{L O}\right)$ differs in the passive and the Gilbert mixer, which are derived in the following sections. Considering the quadrature mixer and assuming that the amplitudes of the LO signal of the I and Q phase are $A_{L O I}$ and $A_{L O P}$, the IBR can be calculated by

$$
I B R=20 \log \left(\frac{V_{I}+V_{Q}}{V_{I}-V_{Q}}\right),
$$

where $V_{I}$ and $V_{Q}$ are the first harmonics of the output signals of the I and Q phase.

\subsection{Image Rejection in Passive Mixers}

A double balanced passive CMOS mixer is shown in Fig. 2(a). Each of the NMOS transistor is working between the 'on' and 'off' states by applying the LO signal to its gate. In the 'on' state, the transistor's drain-to-source impedance is the low drain-to-source channel resistance, denoted as $R_{o n}$. In the 'off' state, the drain-tosource impedance is the drain-to-source capacitor, $C_{o f f}$, assuming the channel resistance is rather high in this case. Ideally, the switches work in the linear region, and the $R_{o n}$ can then be easily calculated as,

$$
R_{o n}=\frac{L}{\mu_{n} C_{o x} W\left(V_{g s}-V_{T}-V_{d s}\right)},
$$

where $\mathrm{L}$ is the gate length, $\mu_{n}$ is the average electron mobility in the channel, $C_{o x}$ is the gate oxide capacitor per unit area, and $\mathrm{W}$ is the gate width. Assuming the RF port is loaded with impedance $Z_{L}$, a $300 \mathrm{fF}$ capacitance, when the switches are on, the output voltage is

$$
V_{\text {out }}=\frac{Z_{L}}{Z_{L}+R_{\text {on }}} \cdot V_{\text {in }} .
$$

It is straightforward that any change in the amplitude of the LO signal leads to a change in the output signal $V_{\text {out }}$ through the on resistance $R_{o n}$. Therefore, the amplitude error in the LO signal is transferred into the output.

With (4) and (5), the first order harmonic of the output signal can be calculated by

$$
V_{1 s t}=\frac{1}{T}\left(\int_{0}^{\frac{T}{2}} V_{\text {out }} \cdot e^{-j \omega t} d t+\int_{\frac{T}{2}}^{T}-V_{\text {out }} \cdot e^{-j \omega t} d t\right) .
$$

\subsection{Image rejection in Gilbert Mixers}

The Gilbert mixer is shown in Fig. 2(b). The mixer is made of a differential trans-conductor, formed by M1 and M2, and a pair of mixer cores, formed by M3-M4 and M5-M6. Each mixer core is a differential pair driven by the input differential LO signal.

When the overdrive voltage $\left(V_{L O}-V_{T}\right)$ of the mixer core is large enough so that the transistors work in the saturation region, the baseband tail current are fully switched to the output. In the other word, the output current is not any function of the amplitude of the LO signal. When the overdrive voltage is not large enough so that the transistors work in the linear region, the output current depends on the amplitude of the LO signal. The graphical analysis is shown in Fig. 3. The first harmonic of the output can then be calculated as

$$
\begin{aligned}
V_{1 s t} & =\left(\frac { 1 } { T } \left(\int_{A}^{B} I_{\text {out }}\left(V_{L O}\right) \cdot e^{-j \omega t} d t\right.\right. \\
& +\int_{B}^{C} I_{B B} \cdot e^{-j \omega t} d t \\
& +\int_{C}^{D} I_{\text {out }}\left(V_{L O}\right) \cdot e^{-j \omega t} d t \\
& +\int_{D}^{E}-I_{B B} \cdot e^{-j \omega t} d t \\
& \left.\left.+\int_{E}^{F} I_{\text {out }}\left(V_{L O}\right) \cdot e^{-j \omega t} d t\right)\right) \cdot R \\
I_{\text {out }}\left(V_{L O}\right) & =\frac{\mu C_{\text {ox }}}{2} \frac{W}{L}\left(V_{L O}-V_{T}\right)^{2},
\end{aligned}
$$

where $\mathrm{R}$ is the load resistance at the RF port. The $\mathrm{LO}$ voltage at the saturation points $\mathrm{B}, \mathrm{C}, \mathrm{D}$, and $\mathrm{E}$ is calculated by $I_{B}=$ $\frac{\mu C_{o x}}{2} \frac{W}{L}\left(V_{L O}-V_{T}\right)^{2}$. Assuming that there is a change in the amplitude of the LO signal, the output current changes only when the transistors work in the linear region. Except for that, the output current is always the whole tail current, $I_{B B}$.

When the amplitude of the LO signal is large enough so that the transistors work in the saturation region most of the time during one period, the difference between B and B' becomes smaller. Therefore, the input amplitude error is rejected to some extend.

When the input LO signal is very small, the differential pair works in a linear fashion, and the output current depends on the amplitude of the LO signal. The output current is always a function of the LO voltage, and is calculated by (8). Therefore, the amplitude error in the LO signal is converted to the output, according to $(2)$.

The above analysis shows that the Gilbert mixer has a much stronger rejection to the input amplitude error when the LO signal is a large amplitude sinusoidal signal, comparing with a small amplitude sinusoidal LO signal. However, this rejection is limited by the case of using a large square wave. When the LO signal is a large square wave, the input amplitude error has no effect at the output, as long as the amplitude of the square wave is larger than the saturation voltage. 


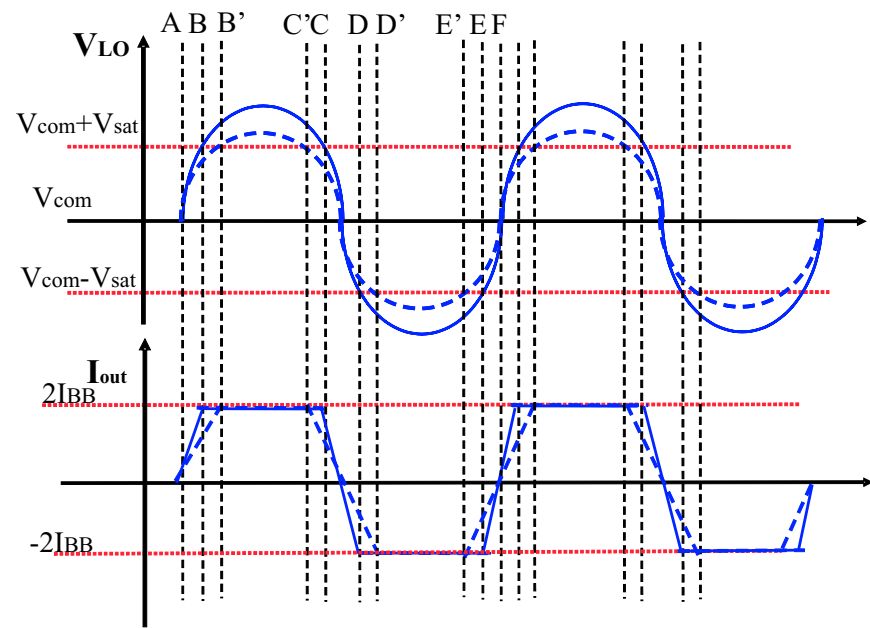

Figure 3: Input and output signal of the active mixer for large LO signal.

\section{COMPARISON OF THE IMAGE REJECTION PERFORMANCE}

In this section, simulated and calculated results are shown to compare the image rejection performance between the Gilbert and the passive mixer.

We consider the case that the mixers are driven by large amplitude sinusoidal signal first. The two circuits were simulated with an input amplitude mismatch between the I and Q phase of the LO signal, $\frac{\varepsilon}{A}$ ranging from $0.1 \%$ to $10 \%$. Equation (6) and (7) are used to calculate the theoretical IBR for the two mixers, respectively. The results are shown in Fig. 4. The input IBR is calculated from (1) with the input mismatch $\frac{\varepsilon}{A}$.

Comparing the simulated IBR in the two mixers, it is shown that both of them present some rejection to the input amplitude error. However, the Gilbert cell shows 10dB stronger rejection than the passive cell, under the same conditions, as expected from the analysis shown in the previous section. Secondly, we compare the simulation and the calculation results for the two mixers. For the passive mixer, the two data matched with each other rather well. For the Gilbert mixer, there is a very large difference between the two values. Further results are shown in the following to find out the reason for this large difference.

The two circuit were simulated with the LO frequency changing from $10 \mathrm{MHz}$ to $2 \mathrm{GHz}$. The $\mathrm{LO}$ signal is still a large sinusoidal signal, and the input amplitude mismatch is set at 5\%. The IBR was calculated again with (6) and (7). All the results are shown in Fig. 5.

We consider the passive mixer first. Both the calculation and the simulation shows that the IBR is independent from the LO frequency. For the Gilbert mixer, theoretically, the IBR is also independent from the LO frequency. However, the simulation results, for the Gilbert mixer, show that the IBR drops very quickly as the LO frequency increases. This seems to be caused by capacitive high frequency effect in the transistors. Further simulations show that the IBR in the Gilbert mixer increases when using smaller transistors for the mixer core. However, this is a tradeoff with the matching issue in the mixer itself. Then we compare the simulated

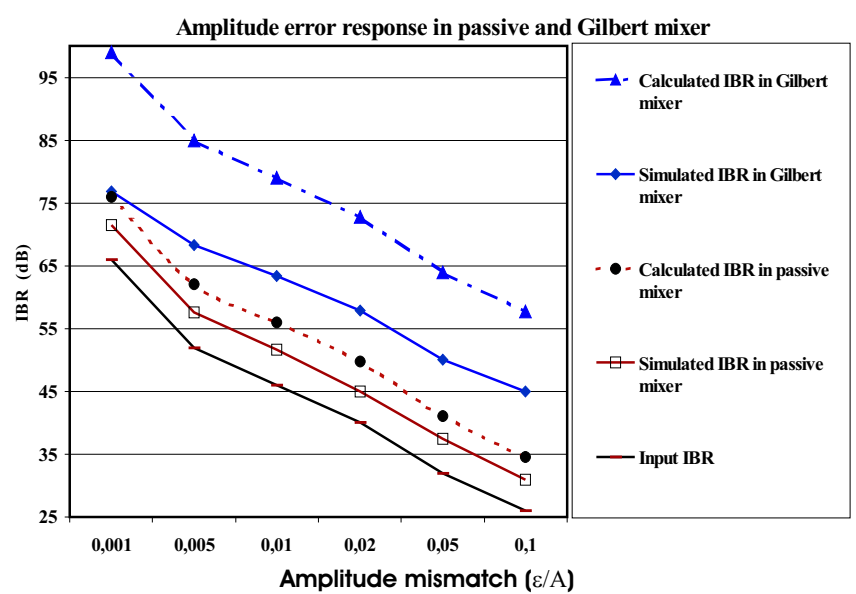

Figure 4: Simulated and calculated IBR for the Gilbert and the passive mixer vs. amplitude error in the LO signal. The amplitude of the $\mathrm{LO}$ signal is $1 \mathrm{~V}$, and the frequency is $2 \mathrm{GHz}$.

image rejection performance of the two mixers, the Gilbert mixer always shows a stronger rejection to the input amplitude error than the passive one. However, it also shows a stronger dependence on the LO frequency.

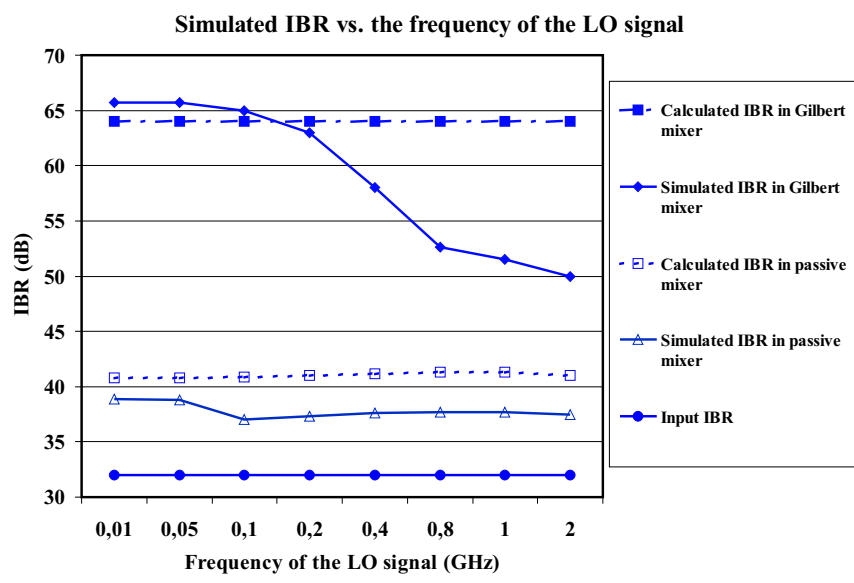

Figure 5: Simulated and calculated IBR for the Gilbert and the passive mixer vs. the frequency of the LO signal. The amplitude of the $\mathrm{LO}$ signal is $1 \mathrm{~V}$, and the amplitude mismatch $(\varepsilon / A)$ is $5 \%$.

So far, all the analysis were done based on a large sinusoidal LO signal. As shown in section II, as the amplitude of the LO signal decreases, the Gilbert mixer shows less rejection to the input amplitude error. In the following part, comparison is made between the two mixers, as a function of the amplitude of the LO signal. Different formulas are used in the calculation for small and large LO signal for the Gilbert mixer. A 5\% amplitude mismatch is assumed at the input, and the $\mathrm{LO}$ frequency is set at $100 \mathrm{MHz}$ in order to avoid the capacitive high frequency effect. All the results are shown in Fig. 6. 


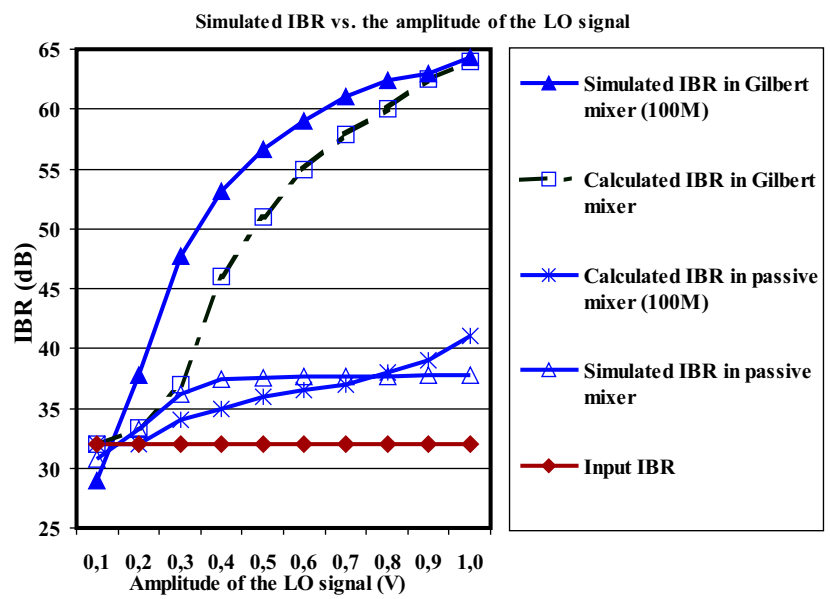

Figure 6: Simulated and calculated IBR for the Gilbert and the passive mixer vs. the amplitude of the LO signal. The frequency of the LO signal is $100 \mathrm{M}$, and the amplitude mismatch $(\varepsilon / A)$ is $5 \%$.

Obviously, as the amplitude of the LO signal decreases, both mixers show less and less rejection to the input amplitude error. According to our analysis, with a very small LO signal, nothing is rejected in the two circuits, as shown in the plot when $V_{L O}$ is $100 \mathrm{mV}$. In general, the Gilbert mixer can reject more amplitude error from the input than the passive cell does. However, this difference becomes less notable when the amplitude of the LO signal gets smaller.

\section{CONCLUSIONS}

A simple model for the image rejection performance in mixers is proposed in this paper, and it is applied to the passive and the Gilbert mixer. The calculations and the simulations match quite well with each other. Comparison of the image rejection performance were done between the passive and the Gilbert mixer. The results show that, with a large sinusoidal LO signal, the active mixer has a stronger rejection to the input amplitude error than the passive mixer does. However, the IBR in the passive circuit has less dependency on the amplitude and the frequency of the LO signal than the its active counterpart has.

\section{REFERENCES}

[1] P. Andreani. "A Low-Phase-Noise, Low-Phase-Error $1.8 \mathrm{GHz}$ Quadrature CMOS VCO”. Proc. ISSCC, pp. 290-291, February 2002.

[2] P. Andreani. "A 2GHz, 17\% Tuning Range Quadrature CMOS VCO with High Figure-of-Merit and $0.6^{\circ}$ Phase Error". Proc. ESSIRC, pp. 815-818, September 2002.

[3] J. Harvey and R. Hariani. "Analysis and Design of an Integrated Quadrature Mixer with Improved Noise, Gain, and Image Rejection”. Proc. ISCAS, pp. 786-789, May 2001.

[4] S. Kang and B. Kim. "Second Order Nonlinearity Analysis of Gilbert Mixer”. Proc. RFIC, pp. 559-562, 2003.
[5] Nam-Soo Kim et. al. "An Image Rejection Down Conversion Mixer Architecture”. Proc. TENCON, vol. 1, pp. 287-289, September 2000.

[6] V. Geffroy et. al. "RF mixers using standard digital CMOS 0.35um process". 2001 IEEE MIT-S Digest, vol. 1, pp. 8386, May 2001.

[7] B. Razavi. RF Microelectronics, Prentice Hall PTR, 1998. 